\title{
THE MORPHOLOGY OF THE PROVENTRICULUS OF A FORMICINE ANT
}

By T. Eisner and E. O. Wilson Biological Laboratories, Harvard University

The ant proventriculus is a structure of considerable entomological interest. It is most elaborately developed in those groups of ants which have the highest form of social behavior and has been generally thought to be associated in some way with food storage and trophallaxis. It has been used extensively as a taxonomic character in the classification of higher categories in the Dolichoderinae and Formicinae and in phylogenetic speculation concerning these groups. Yet, despite several involved descriptions of the anatomy of this organ that have appeared in the past, its function and activity have never been properly understood. The following paper contains the results of a concentrated study of the morphology of the proventriculus of one typical species of formicine. An emphasis has been laid on those details that appear to be relevant to its function, and there is offered a new explanation of its mechanics.

The first accurate anatomical study of the dolichoderineformicine proventriculus was that of Forel (1879). Emery (1888) followed up Forel's work in a classic comparative monograph which is still the basic reference on this organ. He reviewed most of the dolichoderine and formicine tribes, as well as the aberrant myrmicine tribe Cephalotini. Additional brief descriptions of the proventriculus of the Dolichoderinae and Formicinae were given in 1912 and 1925, respectively, in the fascicles of Wytsman's Genera Insectorum dealing with those groups. The most recent research on the formicine proventriculus is that of Forbes (1938), whose account is mostly a review of earlier work and adds little information of significance.

The ant chosen for the present study was Camponotus americanus Mayr. This species proved especially amenable to this type of anatomical work, since it is large and 
possesses a translucent integument. The crop, when filled with stained fluid, can be observed through the gastric tergites, so that those individuals that have their crops distended most suitably for dissection can easily be picked from the artificial nests. China ink was found to be the most suitable of several stains considered for use in studying the course of food in the living gut. This was ground up in honey to make an intensely black liquid, which could be traced even when passing through the fine canal system of the proventriculus. Dissections were accomplished simply by clipping the ant's legs, removing the sternites with a fine pair of scissors, picking out the fat body, and washing the cavity of the gaster with insect Ringer's solution. For histological studies the gut was serially sectioned at seven microns and stained with Ehrlich's haemotoxylin and eosin.

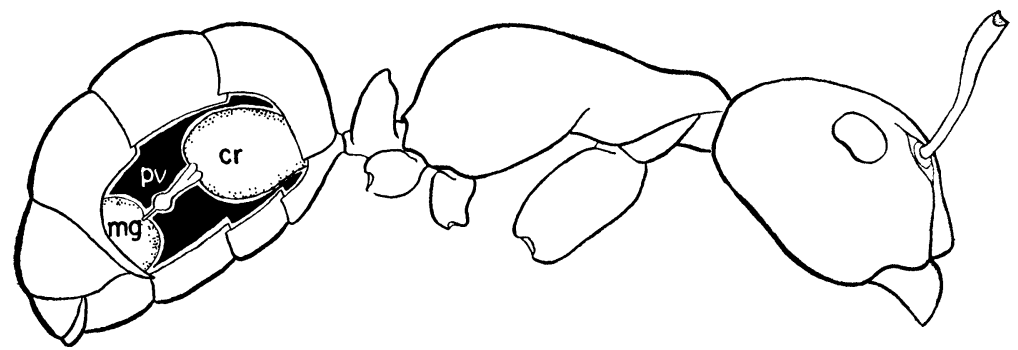

Text-figure 1. Soldier of Camponotus americanus Mayr, with a window cut in the gaster to show size and placement of the proventriculus. $\mathrm{Cr}$, crop; pv, proventriculus; $\mathrm{mg}$, midgut.

The proventriculus, or gizzard, lies in the anterior half of the gastric cavity when not displaced posteriorly by the distended crop (text--fig. 1.). It is the last segment of the foregut and connects the usually voluminous crop with the midgut. In the "euformicine" ants (section

Explanation of Plate 5

Exploded semidiagrammatic drawing of the chitinous framework of the proventriculus. Epithelium and muscularis not shown. A, calyx; B, occlusory tract; C, bulb; D, cylinder. Sep. cnl., sepal canal; filt., filtering slit of sepal; sep., sepal; in., intersepalary intima; cyl. vlv., cylinder valve. (180X) 
PSYCHE, 1952

Vol. 59, Plate 5

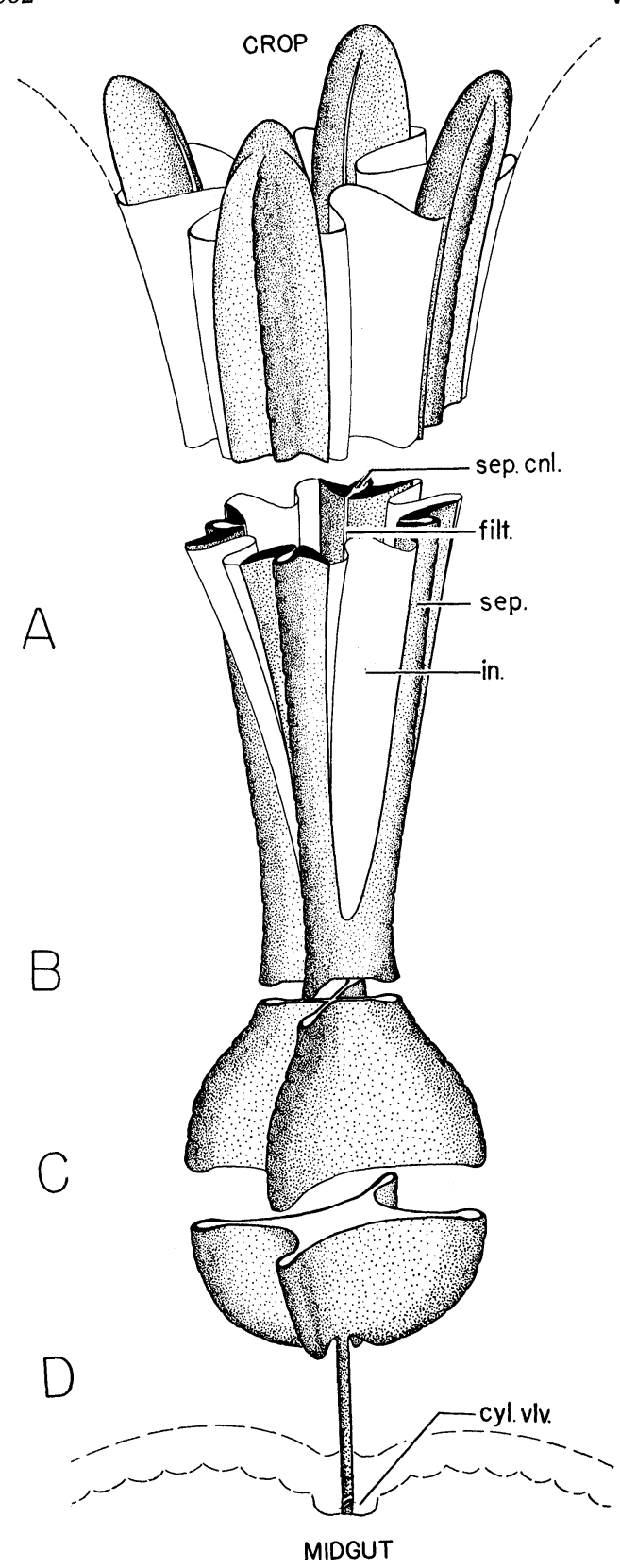


Euformicinae), under which Emery grouped seven of the twelve formicine tribes recognized by him, and including Camponotus, the proventriculus is divided into four distinct parts: the calyx, the occlusory tract ("valve" of Emery), the bulb, and the cylinder (pl. 5; A, B, C, D). The calyx is composed of four strap-like, chitinous rods, or sepals, which extend anteriorly from the body of the proventriculus to capture part of the posterior wall of the crop. It acts like a collapsible funnel which can be closed by the combined action of its intrinsic muscles and the posterior ring muscles of the crop. Seen in cross-section, each sepal is morphologically differentiated into two zones (plate 6, fig. A), which represent two successive phases of secretion by the underlying epithelial cells. The inner area is composed of closely approximated chitinous hairs, which lend it a finely and evenly striated appearance. The outer zone also appears striate, but coarsely and unevenly so, and it is not composed of independent hairs. This area incloses medially the sepal canal, which is to be distinguished from the lateral solid wings of the sepal. The canal opens through a narrow slit to the calyx cavity; this slit is a cross section of the longitudinal cleft which extends the iull length of the sepal. It is guarded by fine, loosely interdigitating chitinous hairs (collectively designated as the fiter), which are actually structural hairs of the inner area of the sepal that have been separated from one another. The canal narrows gradually in the anterior end of the sepal and finally ends blindly; it does not extend into the cavity of the crop. The bulk of the calyx wall is made up of epithelium, designated according to position as sepalary or intersepalary. Externally the wall is limited by a layer of striated muscle, one to two fibers thick, which attaches to the sepals themselves. Between the points of

\section{Explanation of Plate 6}

Fig. A, cross section of the calyx at approximately the level of transection shown in plate 6 . Fig. B, cross section of the occlusory tract at slightly above the level of transection shown in plate 6. Filt., sepal filter; sep. cnl, sepal canal; e’th., intersepalary epithelium; sep. epth., sepalary epithelium; c. mcl., circular muscle fibers; 1 . mcl., longitudinal muscle fibers. (360X) 


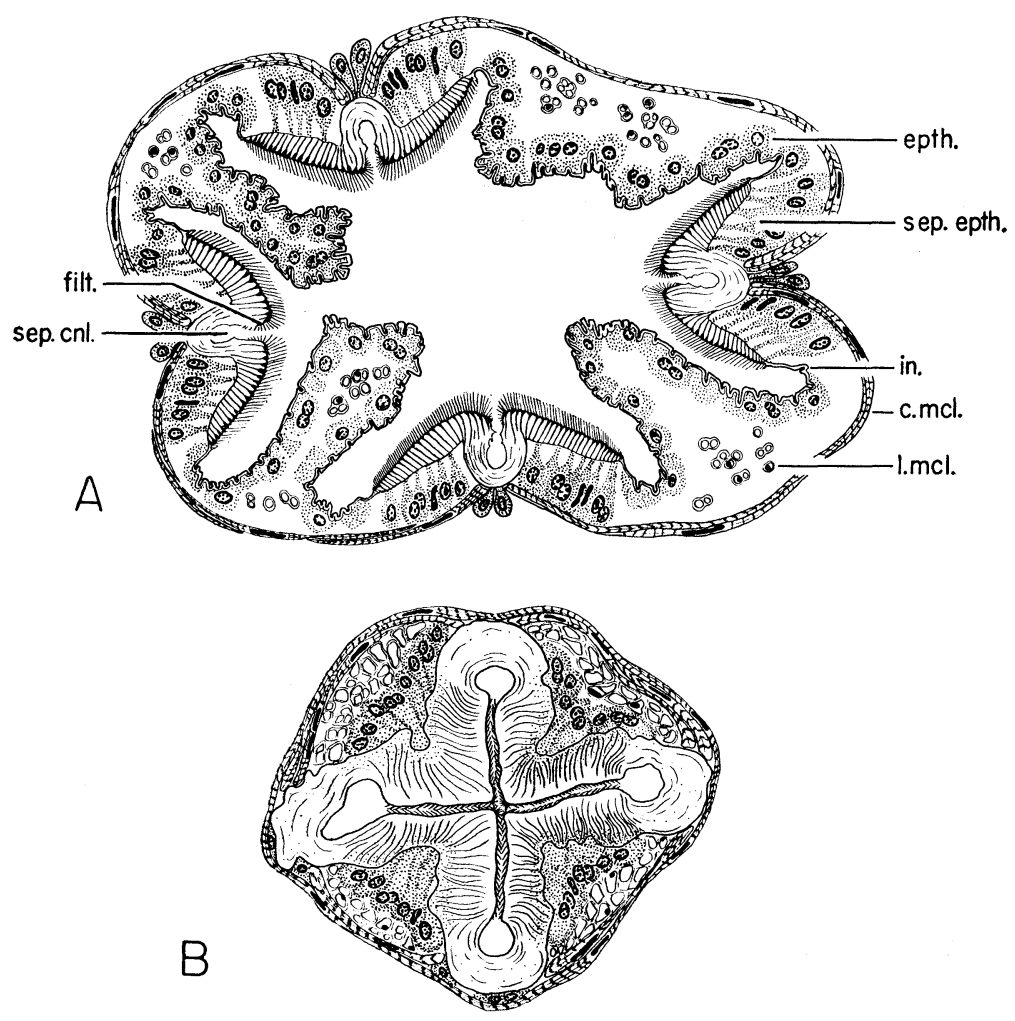

Eisner and Wilson-Formicine Proventriculus 
attachment on each sepal is a pair of external epithelioid cells of unknown function. Internally the calyx wall is lined with a chitinous intima, which is continuous with the intima of the crop wall proper. Scattered in the intersepalary folds are slender longitudinal muscle cells; these are few in number and have no obvious function. They are inserted on the anterior face of the bulb and are continuous with the muscularis of the crop.

The occlusory tract, or valve of the previous literature, is formed posteriorly by a coalescence of the sepals in the following manner. As the calyx narrows, the sepals are brought closer together. Their wings shorten, the inner chitinous zone grows progressively thinner, and the outer zone expands toward the center, extending with it the filter area. Finally the inner zone disappears altogether; the sepals curve together and fuse medially. The filter area, seen in cross section of the sepal as a narrow zone separating the canal from the calyx cavity, has now elongated to form one of the filter slits of the occlusory tract ( $p l$. 6, fig. B). The tract is thus a solid chitinous structure having in the center a single united cruciform slit, the arms of which are expanded at their tips into four canals, which are in turn continuous with the sepal canals in front and the bulb canals behind. It is of interest to note here that the arms of the cross are densely covered with chitinous hairs, whereas the canals are bare. The wall of the tract, external to the chitinous cross, is similar to that of the calyx. The longitudinal muscles are more concentrated due to convergence, and the external pairs of epithelioid cells are no longer evident.

The occlusory tract merges into the bulb by a thinning of the intercanal chitin and an expansion of the cruciform slit into the bulb cavity. The outstanding feature of the bulb is the powerful development of its circular muscles, which are five or more fibers thick in cross section.

\section{Explanation of Plate 7}

Fig. A, cross section of the bulb at approximately the level of transection shown in plate 6. Fig. B, cross section of the cylinder. Fig. C, cross section of the cylinder at the level of the cylinder valve. M. g. epth., midgut epithelium. (360X) 

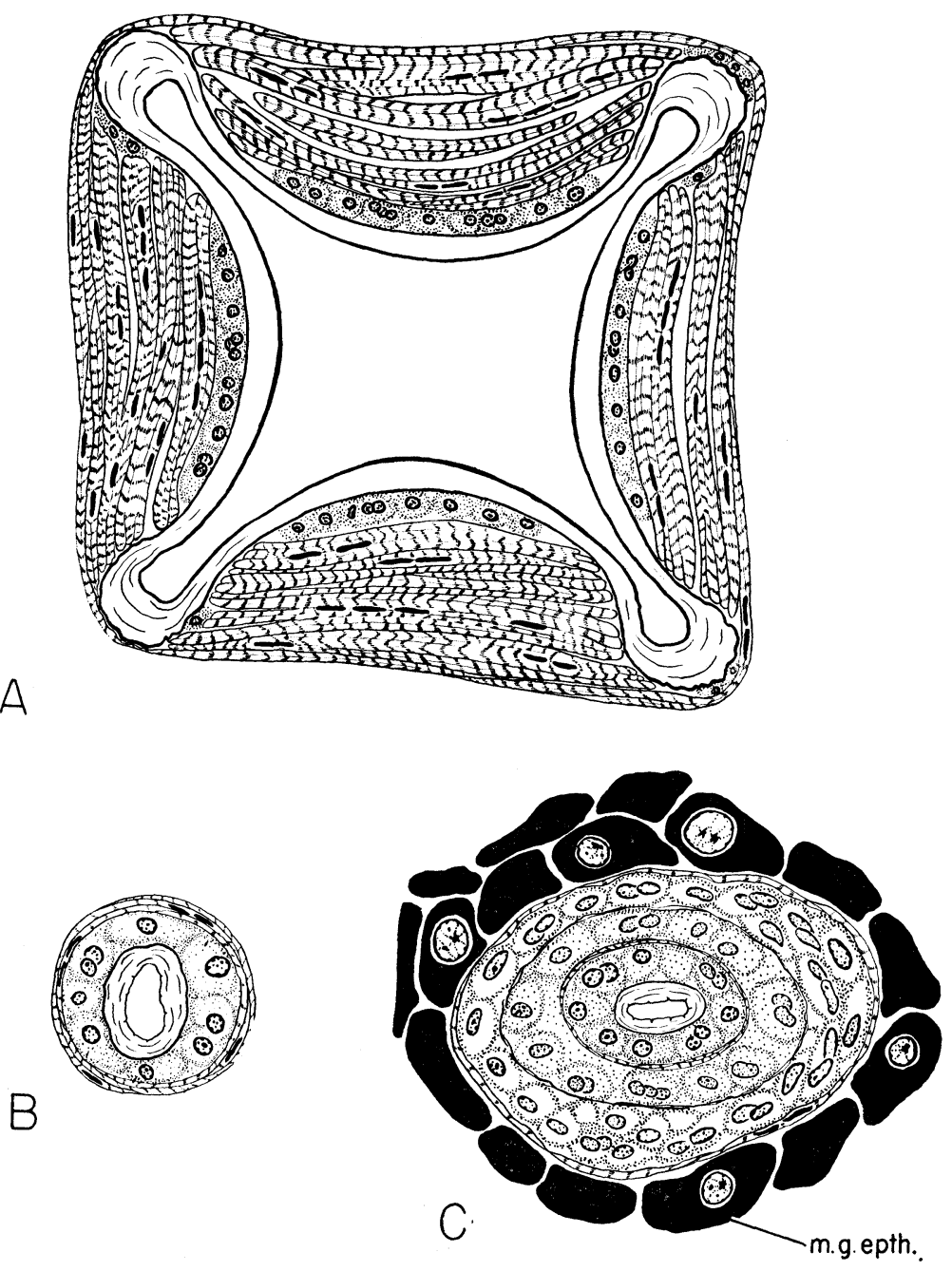

Eisner and Wilson-Formicine Proventriculus 
Longitudinal muscle fibers are absent. Four bulb canals, continuous with those of the tract and calyx, extend the length of the bulb and end blindly at the posterior extremity. In the anterior half of the bulb they do not communicate with the bulb cavity, but are closed off by the approximating chitin walls internally adjacent to them. Posteriorly, the walls diverge so that the canals communicate widely with the lumen when the transverse muscles are relaxed (pl. 7, fig. A), but are closed off from the lumen when the muscles contract. As in the calyx and tract, the outer surfaces of the walls form the points of attachment of the transverse muscles.

The bulb merges into the cylinder by a posterior constriction and a termination of the bulb canals. The circular muscles are again reduced to a thickness of one to two fibers, and there is still no evidence of longitudinal muscle fibers (pl. 7, fig. B). Internally the cylinder consists of a simple chitinous tube surrounded by a single-cell layer of epithelium. At its posterior end, as it enters the midgut, the cylinder is seen in our preparations to fold upon itself in such a way as to become a compound tube (pl. 7, fig. C) . This is made possible by an extreme thinning of the intima in that area. At the end of the cylinder the intima is folded inside out and back upon the cylinder for a short distance; it is then turned inward again and envelops the cylinder as a third fold before it terminates at the midgut junction. The median intima shown in our figure is therefore actually the true intima folded upon itself. The significance of this rather complicated situation is that the tip of the cylinder is highly flexible, allowing the cylinder to be intruded far into the midgut as the cylinder valve ("knob" of previous literature) or to be pulled out of the cavity altogether as a straight, simple tube. Thus the relative lengths of the cylinder and cylinder valve, or those parts outside and inside the midgut cavity respectively, can vary considerably, and the cylinder valve can disappear entirely under some conditions. This phenomenon was first observed by Forel in Conomyrma pyramica (1929) ; we have seen it in several formicine genera, including Camponotus, Lasius, and Prenolepis.

The midgut is of sufficient histological interest to deserve 
a note in passing. The wall consists of a loose aggregation of epithelial cells with variably polyploid nuclei. Our method of fixation (Schaudinn's, five minutes) shows a dense concentration of basophilic granules in the cytoplasm. The cells themselves are so loosely connected that they can be dissected out individually with a fine pin, and it seems unlikely that they are able to withstand much pressure from the contents of the lumen.

It has been generally understood that the proventriculus functions in some way to allow storage of liquid in the crop and to regulate the amount which passes into the midgut. This increase in efficiency at storing food in turn facilitates a greater rate of trophallaxis, which is a fundamental bond of social life in higher ants. As to the specific mode of action of this organ, Emery proposed a scheme in his original paper (1888) which to the present time has been varied upon only slightly. This scheme can be summarized as follows. The occlusory tract acts as a valve which is controlled by the muscles surrounding it and which is able to seal off completely the bulb from the crop. In the euformicines the calyx acts as an accessory valve, collapsing its walls together to prevent the flow of liquid through it. The bulb acts as a unidirectional pump with a valve on each end. As the transverse muscles contract, the adjacent walls of the bulb collapse on one another, first at the periphery and then toward the center, forcing the contents of the cavity down through the cylinder and into the midgut. At the same time the occlusory tract closes off independently and prevents flow of the bulb's contents forward into the crop. As the bulb muscles relax, the elasticity of the chitinous wall opens the cavity and creates suction, while the occlusory tract opens and allows liquid from the crop to fill the bulb. The "knob", the end of the cylinder which enters the midgut cavity, acts as a valve to prevent flow of liquid back from the midgut, since its cavity is collapsed by slight external pressure. Regurgitation is initiated by a closure of the occlusory tract (and calyx also in the euformicines) and an expansion of the pharyngeal cavity; the latter process sucks liquid forward out of the crop. This original scheme was based entirely on prepared, sectioned material. 
Our studies indicate that while the function attributed to the proventriculus by Emery and others is correct, the accepted explanation of its mechanics as given above is basically erroneous. The reason for this lies in several fundamental misconceptions established by Emery in his 1888 paper and maintained in the literature ever since. First, Emery believed that the filter hairs of the euformicine sepal canals actually seal off the canals from the calyx cavity: "Die Rinne wird gegen das Lumen des Kelches durch das Ineinandergreifen der die Flügel besetzenden Härchen verschlossen". Because of this he was inclined to treat the canals as relatively unimportant structures. Subsequent workers, including Forbes, have observed that the canals really open into the lumen, but none have considered the obvious possibility that they might serve to conduct liquid past the occlusory tract. That this conduction may occur has been ascertained by our observations on the proventriculi of Camponotus fed with stained honey. Following spontaneous contractions of the calyx and ejection of the contents into the crop, the canals could often be seen to remain full of the honey, usually back to the level of the occlusory tract, but in one instance through most of the length of the bulb as well. In these cases there were no evidences of the honey in the bulb cavity itself.

A second misconception which Emery established is that the occlusory tract acts as a valve. Actually, even if the occlusory tract could be closed entirely, which is possible, the presence of strongly reinforced canals passing through the tract prevents it from stopping flow altogether. At the same time, the cruciform slit cannot be opened to an extent much greater than that shown in plate 6 . In all, the entire structure of the tract appears to be too rigid to be able to exert much control over the rate of flow by the action of its intrinsic muscles.

A third misconception is that the canals of the bulb are collapsed as the transverse muscles contract. We have already seen that these canals are sealed off in the anterior portion of the bulb even when the muscles are relaxed. It is probable that they become sealed off but not collapsed in the posterior portion as contraction is initiated. Why this happens can be seen in an examination of the cross 
section of the bulb (pl. 7). The walls of the canals are constructed along their outer edges of exceptionally thick chitin, which cannot easily be folded in. The action of the transverse muscles is obviously to decrease the volume of the bulb. The first thing that must occur as this constriction begins is that the opposing chitinous walls immediately adjacent to the canals meet, although the canals themselves remain open. The point of contact of the walls now proceeds toward the center of the bulb, until, at the completion of contraction, the bulb cavity is mostly obliterated.

A fourth misconception, not entirely referable to Emery, but partly stemming from other authors such as Janet, is that the crop is a passive organ which functions only as a storage center. Examination of entire and dissected Camponotus, the crops of which were distended with stained honey, showed that the crop wall is capable of strong and almost total contraction, which at the very least would suffice for regurgitation by itself. In dissected material the movements were most marked in the posterior ring muscles, but they often proceeded anteriorly to include the entire crop. In fully intact but excited and active individuals the crop, when only slightly distended, could be seen through the body wall to undergo very swift pulsations, forcing almost its entire contents into the forward part of the gut.

Our interpretation of the action of the proventriculus in Camponotus americanus is as follows. The calyx and occlusory tract are devices for checking the posterior flow of liquid as fluid pressure builds up in the crop. They are able to do this without muscle contraction, simply because more pressure is required to force liquid through the filtering slits than is present even when the crop is considerably distended. Muscle action can have little effect on the width of these filtering slits and therefore cannot exert a valve-like control per se. In the euformicines the muscularis can act as a secondary damming device by closing the calyx and preventing liquid from reaching the filtering slits. But in dissected individuals with distended crops the closure of the calyx was not a necessary device, and liquid from the crop failed to reach the bulb even when the calyx was open and its cavity filled. The pressure re- 
quired for passing liquid through the calyx and tract is provided by the bulb. As the bulb starts its contraction, its four canals are sealed off, perhaps imperfectly in the posterior portion, but at least completely enough to act as a valve to prevent most of its contents from flowing back into the calyx lumen. As the bulb cavity collapses under muscle contraction, the contents are forced back through the cylinder and into the midgut. As the muscles relax and the cavity expands due to the elasticity of its chitinous framework, the canals of the posterior portion communicate again with the lumen and allow liquid from the anterior canal system to pour in. Probably some of the liquid is sucked in through the filtering slits of the occlusory tract also, but the body of the tract is so extensive and its walls so densely covered with hairs that traffic through it must be relatively insignificant. A forward flow of liquid from the midgut is prevented by the cylinder valve, the thin walls of which can collapse under external pressure.

As we presently understand it, the significance of the characteristic elaboration of the dolichoderine-formicine proventriculus is that it allows the crop to store large amounts of liquid. As previous authors have suggested, it regulates the amount of this liquid which passes into the midgut and in this way separates the individual supply of food from the communal supply. In the concept presented here, it is considered that the primary feature of the advanced proventriculus is the filter area, while the other structures are developed supplementarily. This area acts as a plug guarding the midgut, and liquid can be forced past it only under pressure from the bulb. As its name implies, it also acts as a very thorough filter, and perhaps correlated with this is the fact that the midgut wall is so constructed as to be able to handle only small amounts of pure liquid.

The calyx of the euformicines represents an extension of the occlusory tract which allows a greater filtering area and possibly provides a secondary valvular control by its ability to collapse and occlude liquid from its lumen. In noneuformicine types the calyx is either absent or does not function in this capacity, and the occlusory tract is more massive. In the section Alloformicinae of Emery (Melo- 
phorini, Plagiolepidini, and Myrmelachistini), the occlusory tract is a prominent dome which surmounts the bulb. It is traversed by the same cruciform slit found in the euformicines. Anteriorly the slit is covered by dense chitinous hairs; this area is exactly comparable, if not homologous, to the filtered slits of the euformicine calyx and tract. Posteriorly the slit is devoid of hairs, and the tips of its arms broaden into canals which open into the bulb canals, again very much as in the euformicines. The proventriculus characterising Emery's section Heteroformicinae (Myrmecorhynchini and Myrmoteratini) appear from Emery's figures to be essentially similar to the alloformicine type, and there seems to be little justification for considering them other than aberrant alloformicines. The advanced dolichoderine type of proventriculus (Tapinomini) is the most complicated and difficult to understand, but in essential details it is still similar to the alloformicine type. There is an anterior filter area and posterior canals. Sepals, or sepal-like structures, are present and reflexed back over the bulb, with which they fuse. The chitinous septa produced by the fusion of the bulb and inverted sepals serve as the points of attachment of the bulb muscularis. The proventriculus of the myrmicine tribe Cephalotini has unquestionably evolved independently of that of the Dolichoderinae and Formicinae. Yet here again an occlusory tract abuts the crop and is the principal feature. The filtering slits are irregularly branched; they open into a rather simplified muscular portion which is little different from the primitive proventriculus of other myrmicines.

We wish to express our thanks to Dr. A. B. Dawson and Dr. W. L. Brown for critically reading the manuscript and offering many helpful suggestions.

\section{REFERENCES Cited}

EMERY, C.

1888. Ueber den sogenannten Kaumagen einiger Ameisen. Z. Wiss. Zool., 46:378-412, pls. 27-29.

1912. Formicidae, subf. Dolichoderinae. Wytsman's Genera Insectorum, fasc. 137:1-50.

1925. Formicidae, subf. Formicinae. Ibid., fasc.183:1-302. 
FORBES, J.

1938. Anatomy and histology of the worker of Camponotus herculeanus pennsylvanicus deGeer (Formicidae, Hymenoptera). Ann. Ent. Soc. Amer., 31:181-195.

FOREL, A.

1878. Études myrmécologiques en 1878 (première partie) avec l'anatomie du gésier des fourmis. Bull. Soc. Vaudoise Sci. Nat., 15:337-392, pl. 23.

1929. The social world of ants. English translation, by C. K. Ogden. Albert and Charles Boni, New York. Vol. 1:84-85. 

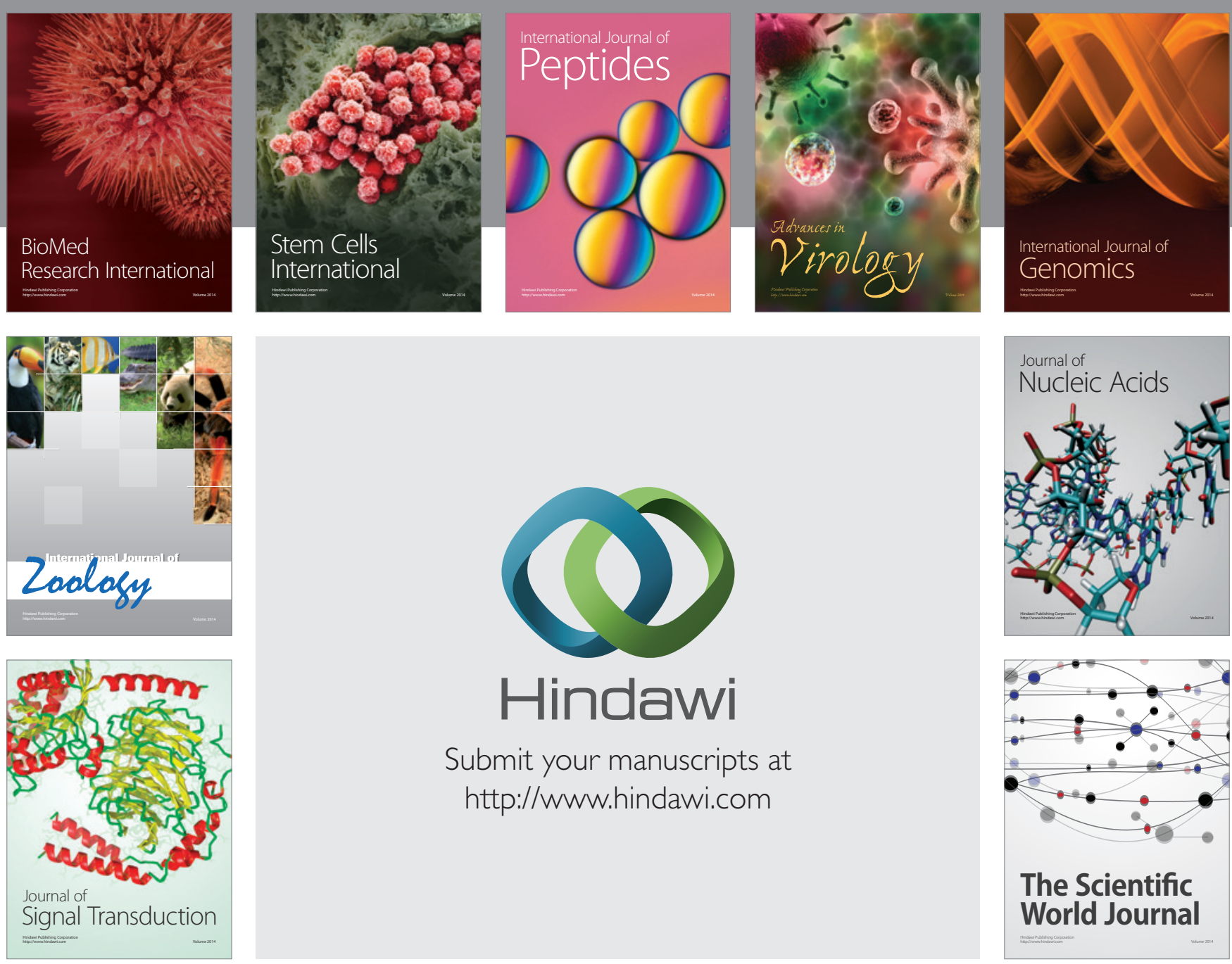

Submit your manuscripts at

http://www.hindawi.com
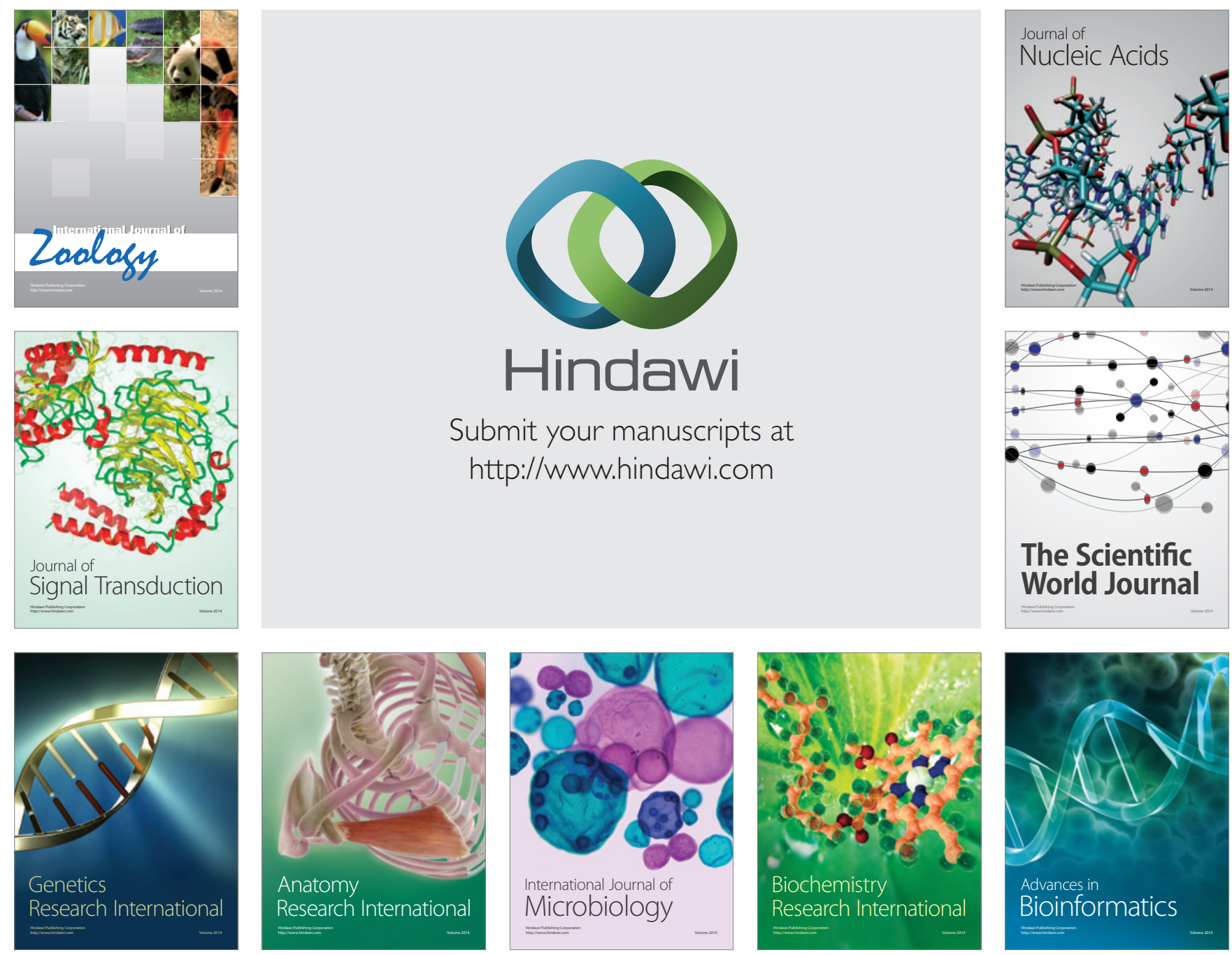

The Scientific World Journal
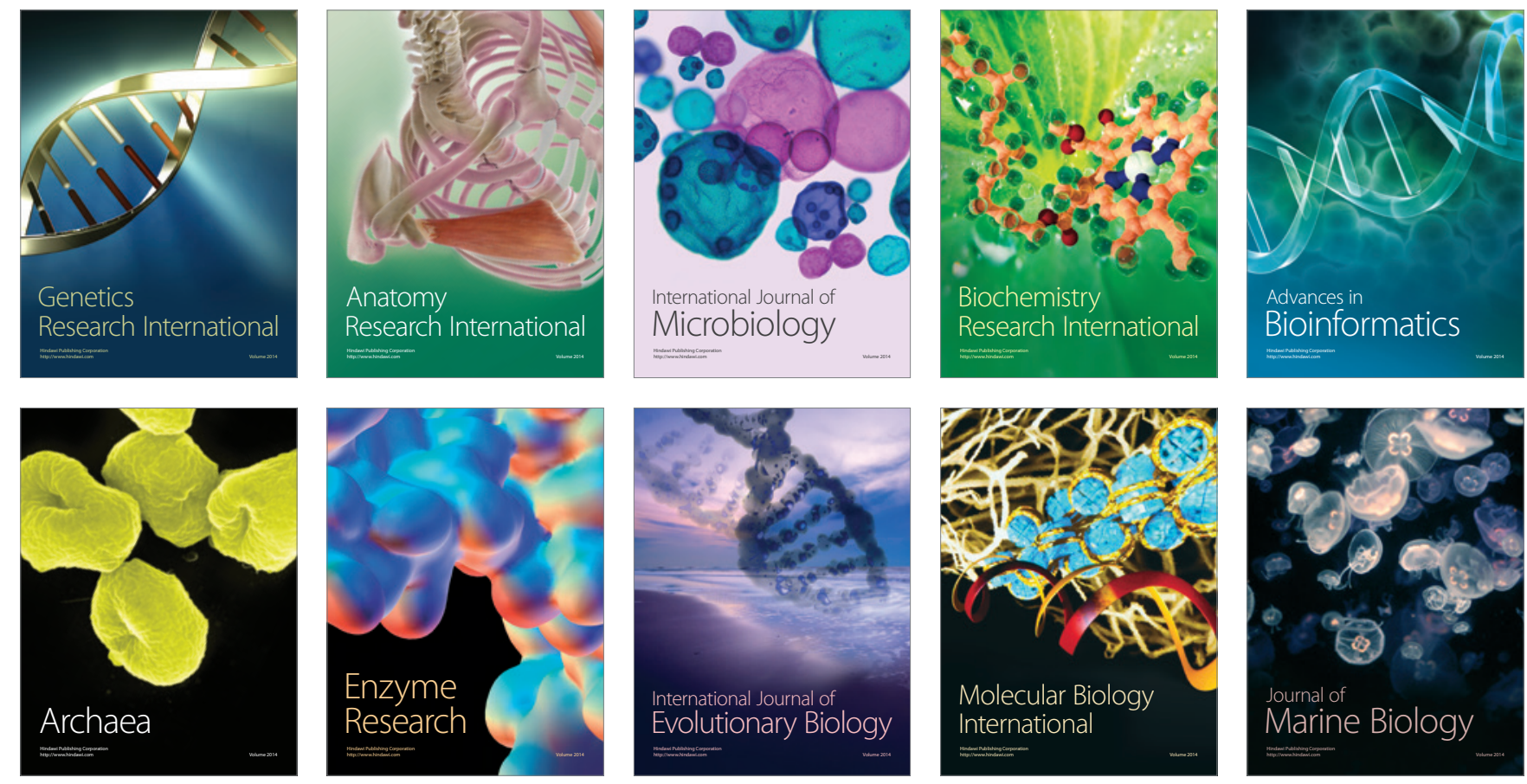